\title{
A150 ADOPTIVE CELLULAR GENE THERAPY OF AUTOIMMUNE ARTHRITIS USING A COMBINATION OF LENTIVIRALLY TRANSDUCED DENDRITIC CELLS
}

U Purath, R J Creusot, C Garrison Fathman, U Müller-Ladner, E Neumann, I H Tarner Department of Internal Medicine and Rheumatology, Justus-Liebig-University Giessen, Division of Rheumatology and Clinical Immunology, Kerckhoff-Klinik, Bad Nauheim, Germany

\subsection{6/ard.2010.129650a}

Purpose In order to minimise systemic side effects of immune-modulating therapy in rheumatoid arthritis (RA), local delivery of anti-inflammatory molecules to the inflamed joints would be desirable. Immune cells are promising vehicle cells for site-specific gene transfer because of their ability to migrate into inflamed tissues such as arthritic joints. In previous studies we have shown that gene transfer of single anti-inflammatory molecules by genetically modified lymphocytes prevents the onset of arthritis in collagen-induced arthritis (CIA) but does not ameliorate established disease despite site-specific homing. We therefore hypothesised that the combined transfer of anti-inflammatory molecules would be more effective. Dendritic cells (DC) were chosen because they are potent antigen-presenting cells that modulate inflammatory responses when expressing anti-inflammatory molecules.

Methods DC were isolated from murine bone marrow by magnetic bead-separation of $T$ cells, B cells and granulocytes cultured in the presence of interleukin 4 (IL4) and granulocyte-macrophage colony stimulating factor (GM-CSF) and transduced using three different lentiviral bicistronic constructs encoding GFP and IL12p40 (DCp40), anti-TNF scFv (DCTNF) or human galectin-1 (DCGal-1), respectively. Different dual (DCp40+DCGal-1, DCp40+DCTNF, DCGal$1+\mathrm{DCTNF})$ and triple (DCp40+DCGal-1+DCTNF) combinations of transduced cells were adoptively transferred into DBA/1 mice after the onset of clinical arthritis. Clinical disease severity, histological severity (pannus formation, cartilage degradation and bone erosion), local cytokine expression were analysed and DC migration was examined histologically.

Results Transduced DC were found to migrate into the inflamed paws. However, the in vivo life span of these cells remains unclear. The combined transfer of DCp40+DCGal-1 resulted in a significant reduction of pannus formation and bone erosion, whereas the other combinations did not. Clinically, there was a trend towards less disease severity in this treatment group which did not reach significance. Local expression of IL10 in the paws was increased in the DCp40+DCGal-1 group whereas interferon gamma was reduced and tumour necrosis factor unaltered. Splenic expression of all three cytokines was reduced in this treatment group. Of note, triple gene therapy was not superior to the combination of DCp40 and DCGal-1.

Conclusions Lentivirally-transduced DC are able to migrate into inflamed joints in CIA. The combined transfer of IL12p40 and galectin-1 has anti-destructive effects and shifts the local cytokine expression towards a Th2 pattern, while clinical efficacy is limited. Routine application is impeded by 


\section{EWRR abstracts}

the inherent complexity of the technique, the variability of gene transduction/expression and the uncertain duration of DC survival. We conclude that the dosage reached was too low in relation to the massive inflammatory activity in our model. 